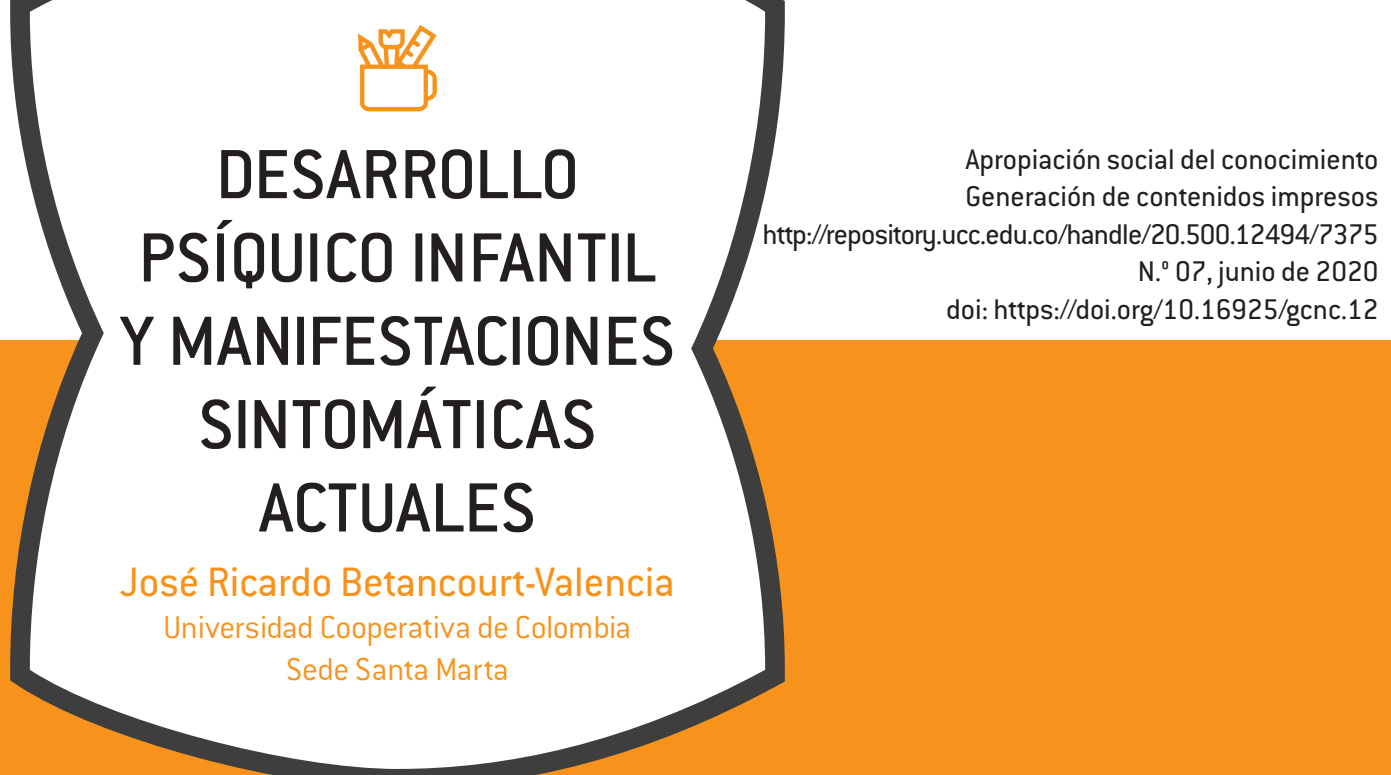

El presente documento de trabajo ha sido incluido dentro de nuestro repositorio institucional como Apropiación social de conocimiento por solicitud del autor, con nes informativos, educativos o académicos. Asimismo, los argumentos, datos y análisis incluidos en el texto son responsabilidad absoluta del autor y no representan la opinión del Fondo Editorial o de la Universidad.

\title{
DISCLAIMER
}

This coursework paper has been uploaded to our institutional repository as Social Appropriation of Knowledge due to the request of the author. This document should be used for informational, educational or academic purposes only. Arguments, data and analysis included in this document represent authors' opinion not the Press or the University. 


\section{ACERCA DEL AUTOR}

José Ricardo Betancourt-Valencia, magíster en Psicología, especialista en Psicología Clínica. Profesor auxiliar de la Facultad de Psicología, Universidad Cooperativa de Colombia, sede Santa Marta, Colombia.

Correo electrónico: jose.betancourt@campusucc. edu.co

ORCID: https://orcid.org/0000-0003-0901-0478

Google Scholar: https://scholar.google.com/citations? user=qk-Lm4QAAAAJ\&hl=es

\section{Cómo CITAR ESTE DOCUMENTO}

Betancourt-Valencia, J. R. (2020). Desarrollo psíquico infantil y manifestaciones sintomáticas actuales (Generación de contenidos impresos N. ${ }^{\circ}$ 07). Bogotá: Ediciones Universidad Cooperativa de Colombia. doi: https://doi.org/10.16925/gcnc.12

Este documento puede ser consultado, descargado o reproducido desde nuestro repositorio institucional (http://repository.ucc. edu.co/handle/20.500.12494/7369) para uso de sus contenidos, bajo la licencia de Creative Commons Reconocimiento-No Comercial-Sin Obra Derivada 4.0 Internacional. http://creativecommons.org/licenses/by-nc-nd/4.0/

\section{(ㄷ) (1) $(9)$}




\section{TABLA DE CONTENIDO}

INTRODUCCIÓN

Propósito del curso y competencias 5

Contenidos temáticos 5

Recomendaciones prácticas sobre las notas de clase 5

UNIDAD 1

$\begin{array}{ll}\text { PREFACIO } & 7\end{array}$

LA CLÍNICA FREUdiAna, ALGUNOS ELEMENTOS BÁSicos 7

¿Qué nos plantea la clínica freudiana clásica? 7

REFERENCIAS II

UNIDAD 2

$\begin{array}{ll}\text { PREFACIO } & \text { I } 2\end{array}$

TRANSFORMACIONES Y NUEVOS MODOS DE PRESENTACIÓN SINTOMÁTICA ACTUAL I 2

$\begin{array}{ll}\text { REFERENCIAS } & \text { I4 }\end{array}$

$\begin{array}{ll}\text { APARTADO FINAL } & \text { I5 }\end{array}$

$\begin{array}{ll}\text { Actividad evaluativa } & 15\end{array}$

\section{ÍNDICE DE FIGURAS Y TABLAS}

Figura 1. Etapas de la estructuración subjetiva

Tabla 1. Cuadro comparativo de etapas de estructuración psíquica y su evolución 


\section{DESARROLLO PSÍQUICO INFANTIL Y MANIFESTACIONES SINTOMÁTICAS ACTUALES \\ José Ricardo Betancourt-Valencia}

\section{RESUMEN}

La nota de clase que a continuación se presenta tiene como intención proporcionar elementos fundamentales para ubicar las transformaciones y manifestaciones contemporáneas de la sintomatología infantil, a partir de un recorrido breve por las etapas del proceso de estructuración psíquica: etapa oral, anal y fálica, y a partir de lo que a nivel subjetivo se juega en cada una en términos de desarrollo. Para posibilitar el logro de la competencia, la presente nota se divide en dos apartados: en el primero se expone lo clásico de la clínica y en el segundo, lo contemporáneo. Con estos dos elementos, el estudiante puede tener una visión amplia para el abordaje psicodiagnóstico de niños y adolescentes considerando lo particular de cada caso para no caer en etiquetamientos totalizadores.

Palabras clave: complejo, psicoanálisis, pulsión, síntoma. 


\section{INTRODUCCIÓN}

\section{PROPÓSITO DEL CURSO Y COMPETENCIAS}

El propósito del curso Optativo I (Clínica de la Infancia y la Adolescencia) se relaciona con la unidad de competencia "Validar las teorías correspondientes al ciclo vital, la psicopatología y la evaluación psicológica para el desarrollo de estrategias dirigidas a la psicoterapia de niños y adolescentes teniendo en cuenta aspectos técnicos e instrumentales de la intervención clínica en estos dos momentos evolutivos". Para cumplirlo, se plantea la necesidad de que el estudiante realice un recorrido por los planteamientos teóricos clásicos de la clínica psicoanalítica, lo cual le posibilita analizar y comprender los desafíos de la clínica contemporánea.

Dentro de la clínica psicoanalítica existen planteamientos teóricos que permanecen y otros que necesariamente han sido modificados en función de las transformaciones sociales, económicas y tecnológicas, lo cual implica un cambio y una transformación en la forma de ver y comprender los fenómenos clínicos de manera que sea pertinente a la hora de intervenir un caso.

Ya sea que la intervención se realice con niños o con adolescentes, es necesario conocer y comprender las transformaciones de la clínica, lo cual proporciona al estudiante una perspectiva respecto a lo que permanece y lo que cambia. También es clave identificar las nuevas formas sintomáticas, con lo cual los clínicos pueden desarrollar una lógica comprensiva de acuerdo con las particularidades de la época contemporánea. Así mismo, se requiere que el clínico pueda leer la época de manera comprensiva para no caer en dogmatismos teóricos estériles. Se espera que el estudiante reconozca cambios y transformaciones en la clínica para dar respuesta a los elementos de competencia: 1) explicar los planteamientos teóricos y epistemológicos del ciclo vital y la psicopatología de la infancia y la adolescencia; 2) juzgar los elementos correspondientes a la evaluación psicológica en la infancia y la adolescencia; y 3) diseñar estrategias dirigidas a la intervención clínica en la infancia y la adolescencia.

Con la intención de generar un proceso de desarrollo de los elementos planteados, la presente nota de clase enfatiza los cambios y las transformaciones de la clínica; y ofrece elementos teóricos para facilitar la identificación de cambios, transformaciones y sus implicaciones en la clínica a la hora de intervenir, así como ejemplos que se pueden observar a diario en el trabajo con niños y adolescentes.

\section{CONTENIDOS TEMÁTICOS}

El documento que a continuación se presenta está conformado por dos líneas de trabajo. La primera tiene relación con lo que serían los planteamientos clásicos respecto al desarrollo infantil y la constitución del sujeto. Esto le permite al estudiante ubicarse en lo que se denomina "clínica clásica".

La segunda línea se desarrolla en cuanto a las transformaciones y los nuevos modos de presentación sintomática, con lo cual el estudiante puede elaborar una visión amplia para la comprensión y el abordaje de dichas problemáticas.

\section{RECOMENDACIONES PRÁCTICAS SOBRE LAS NOTAS DE CLASE}

El documento de la nota de clase, como se mencionó con anterioridad, está dividido en dos apartados. Cada apartado sigue una línea de continuidad que permite aportar al desarrollo de las competencias planteadas.

Es necesario que el estudiante pueda abordar primero, a través de las lecturas sugeridas, lo que es el contexto clásico de la clínica infantil, lo cual le permitirá acercarse de manera 
directa a conceptos y nociones clínicas clásicas para luego llegar a los fenómenos clínicos actuales.

Como estrategia pedagógica, será empleado el comentario a partir de experiencias clínicas; esto con el propósito de que el estudiante pueda ir reflexionando y visualizando el centro de las cuestiones abordadas. Todo lo anterior enmarcado desde el modelo crítico de competencias que ha institucionalizado la Universidad Cooperativa de Colombia, con el cual se busca la integralidad en el conocimiento y la formación profesional, enfatizando la reflexión permanente sobre el saber, el ser y el hacer en un ambiente interactivo de participación y construcción del conocimiento. 


\section{UNIDAD 1}

\section{PREFACIO}

En la Unidad 1, se hará un recorrido breve alrededor de la clínica clásica freudiana, con el propósito de que el estudiante pueda ubicar algunos de los elementos básicos de la clínica infantil y de sus vicisitudes.

De igual forma, se proponen algunas preguntas orientadoras para abrir el panorama. ¿Cuál es la importancia de los aportes de la clínica clásica al abordaje de niños? ¿Qué sentido tiene hacer un recorrido por los planteamientos teóricos de la clínica tradicional? ¿Qué importancia tiene para los psicólogos clínicos reconocer los cambios y las transformaciones de la clínica infantil?

A partir de estas preguntas, se aporta al desarrollo de la unidad de competencia "Validar las teorías correspondientes al ciclo vital, la psicopatología y la evaluación psicológica para el desarrollo de estrategias dirigidas a la psicoterapia de niños y adolescentes teniendo en cuenta aspectos técnicos $e$ instrumentales de la intervención clínica en estos dos momentos evolutivos". Para tal fin, se identifica la relevancia de lo "actual" en las presentaciones sintomáticas contemporáneas y se requiere analizar si es posible sostener los planteamientos teóricos clásicos y su validez actual en el abordaje de niños.

Es necesario que el estudiante haga un recorrido preliminar de los temas a abordar para que se familiarice con algunas nociones clínicas.

\section{LA CLÍNICA FREUDIANA,} ALGUNOS ELEMENTOS BÁSICOS

\section{¿QUÉ NOS PLANTEA LA CLÍNICA FREUDIANA CLÁSICA?}

La clínica freudiana es el punto de partida para todos los desarrollos teóricos y clínicos del psicoanálisis con adultos, niños y adolescentes. Freud nos propone la existencia de ciertos momentos prototípicos en el proceso de constitución subjetiva del sujeto. Para el psicoanálisis, el sujeto no está dado desde el inicio y su desarrollo psíquico tampoco está garantizado, sino que dependerá de las vicisitudes y posibilidades de desarrollo con las que se enfrente.

Estos momentos prototípicos descubiertos por Freud surgieron a partir de sus indagaciones con pacientes adultos neuróticos, principalmente, pero también de algunos acercamientos clínicos directos con niños, como lo evidencian los historiales clínicos en Análisis de la fobia de un niño de cinco años (Freud, 1909). Freud fue identificando cómo en el interior de cada momento prototípico existían algunas fantasías fundantes constitutivas del psiquismo, las cuales —dependiendo de las vicisitudes para su elaboración por parte del sujeto- podían derivar en cierta sintomatología clínica tanto en el niño como en el adulto.

Esta clínica freudiana nos planteaba la sucesión de los momentos prototípicos y la "necesaria ocurrencia" de uno para dar paso a los siguientes y con ello posibilitar la estructuración psíquica del sujeto. Estos planteamientos posibilitaban establecer una progresión más o menos "normal" de lo que ocurría en los primeros tiempos del desarrollo psíquico y en algunas de sus vicisitudes. De igual forma, permitía comprender el surgimiento de cierta sintomatología "característica" de la infancia.

La nosografía freudiana nos proponía como momentos prototípicos de la constitución subjetiva: la etapa oral (destete), la etapa anal (desarrollo del control de esfínteres) y la denominada fase fálica y/o genital (Edipo; Freud, 1905). Estos serían los tres grandes momentos de constitución psíquica para el sujeto, como puede observarse en la figura 1. 


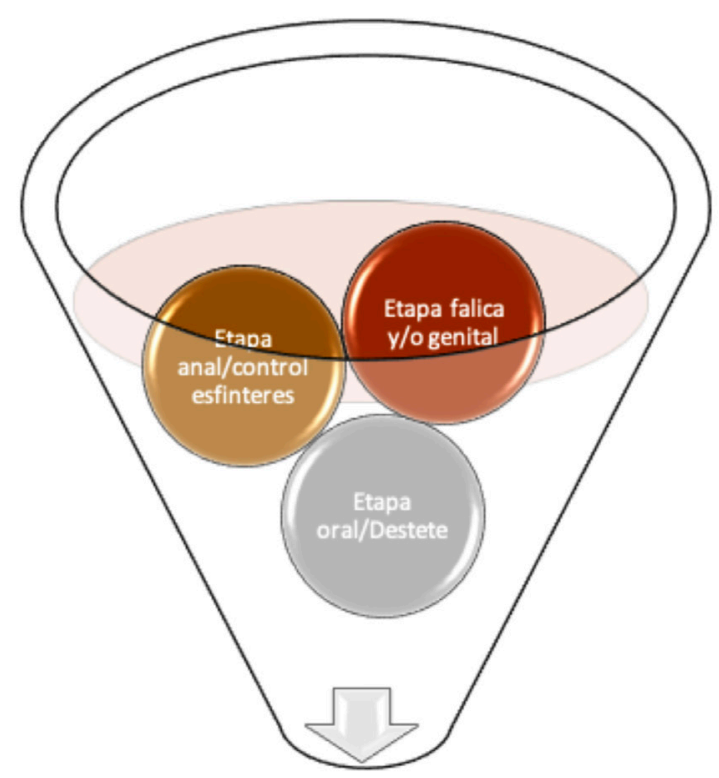

Etapas estructuración subjetiva

FIGURA 1. Etapas de la estructuración subjetiva. Elaboración propia.

Estos tres momentos constitutivos de la estructura subjetiva conformaron las bases y lo "típico" de la clínica. Tal como lo plantea la figura 1, es importante pensar cada momento como tiempos que deben ser atravesados por el individuo para poder convertirse en un sujeto, en los cuales se desarrollan complejos psíquicos (fantasías) como lo es el de destete para la fase oral; como tal, el destete no tiene que ver tanto con el abandono de la lactancia en sí misma, un niño puede haber dejado de ser amamantado, pero no quiere decir que se haya destetado psíquicamente, pues esto requiere otra cosa como operación subjetiva. Esa otra cosa sería el establecimiento de un corte/separación psíquica entre el niño y el otro, que puede ser la madre u otro cuidador.

Otros autores clásicos como Melanie Klein contribuyeron a la comprensión del desarrollo psíquico infantil; según ella, desde el nacimiento el niño es un conjunto de pulsiones desorganizadas y destructivas, la pulsión de muerte estaba presente en el cuerpo del niño y se requería poder sacarla proyectándola fuera del cuerpo. El ser humano requiere poner fuera de sí la pulsión de muerte a través de la relación con el otro; para esto, el otro debe ser vivido como amenazante, lo cual posibilita la constitución de la gran fantasía de la infancia, que es la fantasía de que el otro puede comerme; esto es lo que se pone en juego en el destete, es justamente el atravesamiento de la fantasía de devoración por el otro.

La angustia de ser devorado por el otro es lo propio del tiempo fundacional que se pone en juego en la relación con el otro que cuida al niño. Pero ¿por qué esto se vincula con el destete? Inicialmente, el niño establece con el otro una relación de pura incorporación, el lactante es alguien que incorpora todo el tiempo algo del otro en sus cuidados; sin embargo, llega un momento en el que el niño rechaza al otro, ya sea porque empieza a morder el pezón, ya sea porque corre la cara y no quiere dejarse alimentar, ya sea porque en última instancia vomita, algo que es común entre los 8 y 9 meses.

El primer paso fundacional del destete es el rechazo del alimento, tarde o temprano un niño tiene que poder rechazar el alimento, ese es el primer corte a la alienación del otro. "No me voy a dejar alimentar..." piensa el niño, pero no dejarse alimentar es algo que trae consecuencias, porque rechazar el alimento es en última instancia rechazar al otro; ahí es que se constituye esa fantasía de devoración por parte del otro.

Esta fantasía es central y en los casos en que no se constituye, hoy es muy frecuente encontrar niños que presentan problemas tempranos con la alimentación. En el caso más dramático, podríamos situar dos polos para ubicar estos síntomas en relación con la alimentación. Por un lado, estarían los niños que hacen un rechazo total a la comida u otros casos en los que se desarrolla una fijación a un solo tipo de alimento o a unos pocos. 
Esto puede aparecer incluso en los primeros meses de vida y son conductas que muestran la presencia de procesos defensivos intensos en relación con el cuidador.

Por otro lado, la dificultad asociada al destete puede adquirir la forma de la alimentación compulsiva "bebes comelones, insaciables, golosos", compulsivos. Los niños no nacen con compulsión a la comida, tampoco son omnívoros espontáneamente; si bien es posible que un niño pueda comer muchas cosas inicialmente, luego empezará a dejar de comer algunas de ellas. Esto que en primera instancia puede parecer disfuncional, que puede parecer un problema, es algo que en realidad muestra una operación de corte/separación en la relación con el otro. Es importante resaltar cómo la alimentación durante los primeros tiempos del desarrollo tiene que ver con la relación al otro cuidador y su deseo. Hay padres que se obsesionan y obsesionalizan el comer de sus hijos o cargan de mucha ansiedad la situación del comer.

Aquellos niños en los que no se da el destete de manera temprana, empiezan a vivenciar el destete cuando ya empiezan a comer sólidos, por eso una de las quejas más comunes de los adultos con los niños es que empiezan a jugar con la comida; cuando el niño juega con la comida, es porque descubre el que la comida puede ser para hacer algo más que alimentarse. Entonces, ubicamos esta primera fase que es la del destete, a pesar de lo molesto que pueda ser para los adultos, la función que tiene el destete es la de poder registrar para el niño que el alimento no solamente es para comer, de hecho, el alimento puede ser para jugar, con lo cual se despliega la capacidad de simbolizar y representar diferentes cosas.

Cierta problematicidad en el comportamiento infantil que puede parecer disfuncional, aquella que es muchas veces una molestia para un adulto, es justamente en la que se dan los hitos de desarrollo y crecimiento. Esto nos tiene que llevar a pensar en las dificultades del adulto para soportar el proceso de subjetivación del niño, porque la subjetivación del niño implica cortes en la relación con el otro; implica disfuncionalidad, implica desadaptación; la crianza no se trata únicamente de que los niños se adapten a todas las condiciones planteadas por los padres.

Dado el primer corte en la relación con el otro a partir de la fantasía de devoración, eso implica una posición de gran vulnerabilidad psíquica del niño en la relación con el otro; para ello, es necesario el atravesamiento de un segundo complejo, que es el que llamamos el complejo de control de esfínteres (etapa anal). El complejo del control de esfínteres no tiene que ver solo con el control de las heces, una cuestión que es meramente fisiológica. Controlar las heces no equivale a que hayan atravesado el complejo del control de esfínteres.

Tiene que ver con la posibilidad de estructuración de una segunda gran fantasía de la infancia. Si — como se plantea antes-a partir del atravesamiento del destete el niño queda situado respecto a otro que pueda devorarlo, es necesario que el niño pueda ubicar algún tipo de respuesta que pueda poner algún límite a esa devoración, el cual es precisamente que el niño descubra que pueda dar "algo", que de alguna manera puede ofrecer algo para calmar a ese otro devorador, que puede sacrificar una parte de su cuerpo para calmar a eso otro devorador, y qué mejor parte del cuerpo propio para sacrificar que las heces.

En última instancia, las heces tienen un valor simbólico muy importante $\mathrm{y}$, de hecho, es la gran primera simbolización que se da en la infancia, sobre todo porque permite que el niño pueda establecer una relación con el otro en la que parcializa la angustia de devoración. Por eso Freud decía que las heces tienen la significación psíquica del regalo: "Puedo darte algo a cambio para que no me comas". Este 
fenómeno también se observa en múltiples cuentos infantiles en los que el intercambio es el eje de la historia. El resultado psíquico del atravesamiento del complejo de esfínteres es la separación entre Yo y No yo. El valor de los excrementos es justamente que una parte de mi cuerpo puede separarse y no ser yo, por eso coincide con la consolidación de lo que Freud (1914) llamaba el narcicismo.

Siguiendo en rigor lo que se acaba de plantear, entonces hay una primera aparición subjetiva del niño en el destete a partir de la capacidad de rechazar al otro, de poder decirle no. Ahora, si acabamos de decir que el narcicismo se constituye a partir del complejo del control de esfínteres y que ahí es que aparece el Yo, ¿quién era el que decía no antes de que estuviera el Yo?

Este es uno de los datos más importantes de la concepción psicoanalítica de la infancia; que el sujeto aparece antes que el Yo y que el sujeto aparece primero como una negación, como una capacidad de negar; solo posteriormente esa capacidad de negar es asumida por un Yo. El Yo no está desde el principio, y para que pueda aparecer el Yo, es necesario el complejo del control de esfínteres, que a su vez es fundamental para que aparezca la primera relación simbólica con el otro, porque eso implica la posibilidad de establecer una relación de intercambio con el otro; si yo puedo darte algo, salgo de la lógica de presencia-ausencia, de estar o no estar que eso es propio del complejo del destete.

Algunos ejemplos clínicos para entender mejor el proceso descrito: un niño que vive fuertes angustias de separación, que no puede separarse de sus padres, que hacia los dos años no puede entrar al jardín, no lo pueden dejar en el jardín porque se pone a llorar, es un niño que seguramente simbolizó de manera deficiente la relación con el otro, que no puede contar con la posibilidad de que el otro se ausente porque si esto ocurre, quiere decir que es un niño al que no le alcanza que sus padres le den un beso en la puerta del colegio y que le digan que lo pasan a recoger más tarde porque no cuenta con la presencia del otro; en última instancia, el complejo de destete alcanza para que el niño pueda distinguir entre él y el otro, pero no entre dos posibilidades de él mismo.

Hacia los cuatro años, lo esperable es que un niño ya juegue de manera autónoma, que ya no dependa del adulto, de la mirada del adulto, para poder jugar; sin embargo, ocurre cada vez con más frecuencia que esa simbolización de la mirada del adulto no se dé y que por lo tanto el niño esté cada vez más pegado a la presencia del adulto, lo mismo podríamos pensar respecto a trastornos cada vez más frecuentes en relación con el dormir.

Niños que no pueden dormir solos, por ejemplo, no son niños que lograron dormir solos y que en algún momento, por la aparición de un síntoma, ya no pueden volver a dormir solos; ahí ya deberíamos pensar que algo ya se constituyó y el síntoma viene a romper lo constituido; estamos hablando más bien de casos en los que no se logra constituir la ausencia del otro. En algunos casos, no basta con que el padre se quede en la habitación del niño, es necesario por ejemplo que el padre o la madre se acueste a su lado; aquí es notable el uso del recurso del sentido del tacto, la relación táctil con el otro. El tacto es el único sentido que no es posible desarrollar en ausencia; tocar implica tocar y ser tocado, y es a la vez el sentido más pasivizante; por eso a algunos niños no les es suficiente con que los padres les digan que están en la habitación contigua. No alcanza ni con la mirada ni con la voz, porque donde no se inscribe la ausencia del otro de manera simbólica prima el funcionamiento táctil; son niños que solo se calman de la angustia de separación cuando son tocados. 
Tabla 1

Cuadro comparativo de etapas de estructuración psíquica y su evolución

\begin{tabular}{|c|c|c|c|c|c|}
\hline Etapas & Modo de relación & Complejo & Contenido & $\begin{array}{l}\text { Mecanismo } \\
\text { de defensa }\end{array}$ & Resolución \\
\hline Oral & $\begin{array}{l}\text { Incorporación del otro } \\
\text { - Presencia/ausencia }\end{array}$ & Destete & $\begin{array}{c}\text { Separación/no separación del } \\
\text { otro - Fantasía y angustia de } \\
\text { devoración }\end{array}$ & $\begin{array}{l}\text { Proyección de } \\
\text { lo agresivo }\end{array}$ & $\begin{array}{l}\text { Atravesamiento fantasía de } \\
\text { devoración }\end{array}$ \\
\hline Anal & Intercambio & $\begin{array}{l}\text { Control de } \\
\text { esfínteres }\end{array}$ & Pérdida de algo significativo & Simbolización & $\begin{array}{l}\text { Separación entre Yo y No yo. Dar } \\
\text { algo (heces) para no ser devorado }\end{array}$ \\
\hline Fálica & Rivalidad/sumisión & $\begin{array}{c}\text { Edipo } \\
\text { [castración] }\end{array}$ & $\begin{array}{l}\text { Diferencia sexual - Fálico/ } \\
\text { castrado }\end{array}$ & & Asumir la diferencia sin angustia \\
\hline
\end{tabular}

Nota. Elaboración propia.

La tabla 1 muestra de forma comparativa las diferentes etapas de la estructuración psíquica de los niños y lo que se juega en cada una en cuanto al desarrollo de la subjetividad. Entonces, teniendo en cuenta todo lo planteado, es clara la importancia de reconocer los principales hitos del desarrollo psíquico infantil y sus vicisitudes en términos psicopatológicos.

\section{REFERENCIAS}

Freud, S. (1905). Tres ensayos para una teoría sexual (L. Ballesteros y de Torres, trad.). Biblioteca Nueva.

Freud, S. (1909). Análisis de la fobia de un niño de cinco años (L. Ballesteros y de Torres, trad.). Biblioteca Nueva.

Freud, S. (1913). Pulsión y destinos de pulsión (L. Ballesteros y de Torres, trad.). Biblioteca Nueva.

Freud, S. (1914). Introducción del narcisismo (L. Ballesteros y de Torres, trad.). Biblioteca Nueva. 


\section{UNIDAD 2}

\section{PREFACIO}

La Unidad 2 tiene como propósito exponer algunos cambios en las manifestaciones sintomáticas que se presentan hoy día en la clínica con niños y las implicaciones que esto tiene en cuanto al proceso de evaluación y psicodiágnóstico. De igual forma, se plantean algunas reflexiones en torno a diferentes situaciones que se presentan en la escuela y que permiten que los estudiantes obtengan elementos teóricos para la compresión y el abordaje clínico.

\section{TRANSFORMACIONES Y NUEVOS MODOS DE PRESENTACIÓN SINTOMÁTICA ACTUAL}

Partiendo de los elementos desarrollados a lo largo de la Unidad 1, la clínica freudiana es un punto de partida que permite ubicarnos en torno a lo que fue considerado en su momento como lo prototípico en la infancia. Constituye entonces una referencia ineludible para contextualizar con mayor precisión las transformaciones que se presentan hoy día en niños en cuanto al proceso de constitución subjetiva.

La visión freudiana de la infancia estuvo enmarcada en una época con condiciones sociales e históricas particulares, las cuales han cambiado profundamente y ya no es posible decir que los planteamientos freudianos se sostienen de la misma manera. Hoy, encontramos con mayor frecuencia sujetos con sintomatologías cada vez más difusas en cuanto a su presentación, lo cual dificulta ubicar un diagnóstico estructural del sujeto. Con esto se abre un desafío para el abordaje clínico, puesto que demanda repensar las condiciones en las cuales se desarrollan los niños y su subjetividad en lo contemporáneo.

Se viene notando en la clínica una modificación en los síntomas infantiles. Hasta hace algunas décadas, era común que las consultas estuvieran relacionadas con temores, problemas de aprendizaje y síntomas que de alguna manera inhibían la capacidad de aprender o que limitaban la interacción social del niño. En los últimos años, los motivos de consulta se han ido ampliando, así como los síntomas presentes resultan más inespecíficos, como si de alguna manera hubiese perdido consistencia la noción de síntoma. Para el psicoanálisis, con síntoma (Freud, 1915a) nos referimos a una producción psíquica muy elaborada, compleja, y que implica el proceso de la represión. Según la teoría clásica freudiana, un síntoma puede aparecer hacia los 5 años cuando el niño ha alcanzado la etapa de la latencia o cuando se está atravesando el complejo de Edipo (Freud, 1924).

En la práctica clínica actual, cada vez empiezan a aparecer comportamientos problemáticos más tempranos, esto es, niños de 2 o 3 años en los que los síntomas son más inespecíficos; aparecen síntomas relacionados con trastornos de la alimentación, síntomas enuréticos y encopréticos no reactivos (es decir, cuando el niño no ha alcanzado el control de esfínteres previamente y llega a los 5 años y tiene que usar pañal), niños con trastornos del sueño o niños que nunca llegaron a dormir solos.

Una primera diferencia entre los síntomas clásicos y los nuevos es que los clásicos implicaban la afectación o interrupción de un proceso psíquico ya estructurado; mientras que en la actualidad lo que se presenta son síntomas relacionados con dificultades en la constitución misma, procesos psíquicos muy tempranos que no llegan a desarrollarse y que ponen en suspenso el desarrollo mismo.

Winnicott (1955), otro autor con aportes valiosos para la comprensión del desarrollo infantil, planteaba que uno de los elementos indicadores de la necesidad de tratamiento era la interrupción del crecimiento. Así mismo, planteaba la necesidad de que el entorno 
fuera lo suficientemente bueno para promover y sostener el crecimiento.

Se evidencia en la práctica cotidiana que niños muy pequeños dejan de crecer; ya no se trata del crecimiento interrumpido u obstaculizado por un síntoma, sino por afecciones tempranas del crecimiento.

En Pulsión y destinos de pulsión (Freud, 1913), se establece que hay diferentes destinos para la satisfacción de un niño, así como de los adultos, y ubica a la represión como la forma más elaborada de tratar la pulsión, el instinto sexual. El síntoma sería el resultado de una represión. Freud también ubica otros destinos posibles como característicos de la infancia: el cambio por lo contrario y la uuelta sobre sí mismo; estos mecanismos actúan cuando falta el mecanismo de la represión. Un ejemplo de la vuelta sobre sí mismo está asociado a los "accidentes" que tienen los niños de manera frecuente (golpes, raspones, cortaduras, etc.).

Llama la atención, hoy en día, que pasan los años y esos niños continúan accidentándose. El exceso de exposición a la posibilidad de accidentarse habla de la prevalencia de este mecanismo pulsional temprano. Lo que puede encontrarse en niños de 6 o 7 años que siguen siendo torpes a pesar del crecimiento es que tienen dificultad para establecer el límite de su propio cuerpo y el del otro, son carentes de barreras a su pulsionalidad.

En el caso del otro destino pulsional, el cambio por lo contrario, para Freud el prototipo de esto es el paso del sadismo al masoquismo. Para Freud, en el inicio la pulsión es sádica y está volcada hacia el exterior, y solo por una transmutación se torna masoquista, vuelve sobre la propia persona. Un ejemplo de ello son los niños que "buscan" hacerse castigar todo el tiempo, lo que demuestra que el castigo puede estar fuertemente erotizado en un niño. Formas cotidianas de esto serían la búsqueda permanente de límites; esto se escucha en los padres cuando expresan la "intencionalidad" del niño de hacer cosas a propósito, que parece no acatar normas o reglas y muestra una actitud desafiante provocadora de la respuesta agresiva del adulto. La transgresión de la norma puede tener un valor erótico en la infancia.

¿Qué sucede cuando el fracaso de la represión se torna algo propio de la infancia? ¿Cuándo la represión no llega a establecerse? Teniendo en cuenta que la represión es el mecanismo psíquico que separa lo consciente de lo inconsciente (Freud, 1915b), se encuentran cada vez más niños en quienes esta división no llega a darse; algo que llega a verse en esos niños de 6 o 7 años que continúan siendo muy fantasiosos, que les cuesta distinguir la fantasía de la realidad, y debido a esto se les dificulta prestar atención a las tareas escolares; o en niños que son excesivamente "distraídos" o son "hiperactivos" que no pueden contener sus impulsos y procuran su descarga inmediata sin que al parecer logre mediar el pensamiento o la palabra del otro. Nos encontramos cada vez más no con aquello considerado por Freud como lo habitual, sino con aquello que consideraba como la excepción, procesos mentales arcaicos o que evolucionan a niveles superiores. Esto representa un límite para la teoría y para pensar los casos "nuevos" o una generalización de lo que antes se consideró excepcional.

Los tres momentos de la constitución subjetiva son importantes para comprender las manifestaciones clínicas contemporáneas. Lo que la clínica clásica planteaba en torno al complejo de Edipo como destino del desarrollo del sujeto ya no es lo más frecuente, sino que son síntomas de origen más arcaico. La capacidad de juego se encuentra menoscabada por influencia de las nuevas tecnologías, la falta de conexión de los padres con lo que les sucede a sus hijos o las dificultades para ejercer su función. 
Producto de las transformaciones sociales, económicas, tecnológicas y familiares, hoy en día las escuelas se han convertido en un espacio muy importante, puesto que en la medida en que logren o no acoger las dificultades que se presentan en el desarrollo de los niños, tendrán un papel como ambientes protectores o desestabilizadores del desarrollo.

\section{REFERENCIAS}

Freud, S. (1913). Pulsión y destinos de pulsión (L. Ballesteros y de Torres, trad.). Biblioteca Nueva.

Freud, S. (1915a). La represión (L. Ballesteros y de Torres, trad.). Biblioteca Nueva.

Freud, S. (1915b). Conferencia 23: Los caminos de la formación de síntoma (L. Ballesteros y de Torres, trad.). Biblioteca Nueva.

Freud, S. (1924). El sepultamiento del complejo de Edipo (L. Ballesteros y de Torres, trad.). Biblioteca Nueva.

Winnicott, D. W. (1955). Aspectos clínicos y metapsicológicos de la regresión dentro del marco psicoanalítico. Psikolibro. 


\section{APARTADO FINAL}

Finalizadas las unidades 1 y 2, es importante que el estudiante tenga presente que un adecuado proceso psicodiagnóstico debe considerar lo particular de cada caso para no caer en generalizaciones que borren lo singular de cada sujeto, pues lo pierde de vista como eje fundamental del proceso. Se trata es de comprender al sujeto en su dinámica particular para dar paso a su palabra; más que reducir todo a una categoría diagnóstica silenciadora.

De igual forma, hay que tener presente que los diagnósticos en la infancia deben escribirse con lápiz, lo que equivale a que es posible replantearlos a lo largo del proceso terapéutico.

A continuación, el estudiante debe realizar una revisión bibliográfica para profundizar en el conocimiento de las problemáticas contemporáneas que más se presentan en niños y adolescentes, a fin de contrastarlo con lo planteado dentro de la nota de clase.

\section{ACTIVIDAD EVALUATIVA}

\section{Revisión bibliográfica de problemáticas en} niños o adolescentes: propuestas para la intervención desde la promoción o prevención.

Objetivo general: elaborar una revisión bibliográfica sobre las principales problemáticas en niños y adolescentes que permita profundizar su comprensión a partir del análisis de lo propuesto por diferentes autores.

\section{Objetivos específicos:}

1. Describir y caracterizar psicológicamente una de las problemáticas psicológicas presentes en niños y adolescentes.

2. Desarrollar una fundamentación teórica respecto a la problemática a partir de los artículos.

Metodología: mediante una revisión bibliográfica en bases de datos y revistas indexadas de la universidad, buscar información sobre el tema propuesto. Luego de seleccionados los artículos por su pertinencia, procederán a desarrollar los objetivos 1 y 2.

Referencias: Estilo APA, deben estar completas y guardar relación con las citas en el cuerpo del trabajo.

- Mínimo cuatro artículos por integrante.

- Anexar pantallazos de uso de base de datos UCC.

\section{Puntos por presentar:}

- Hoja de presentación.

- Introducción: Incluye descripción de la problemática (usar citas).

- Fundamentación teórica (toda la información debe ser parafraseada e incluye uso de citas).

- Referencias. 


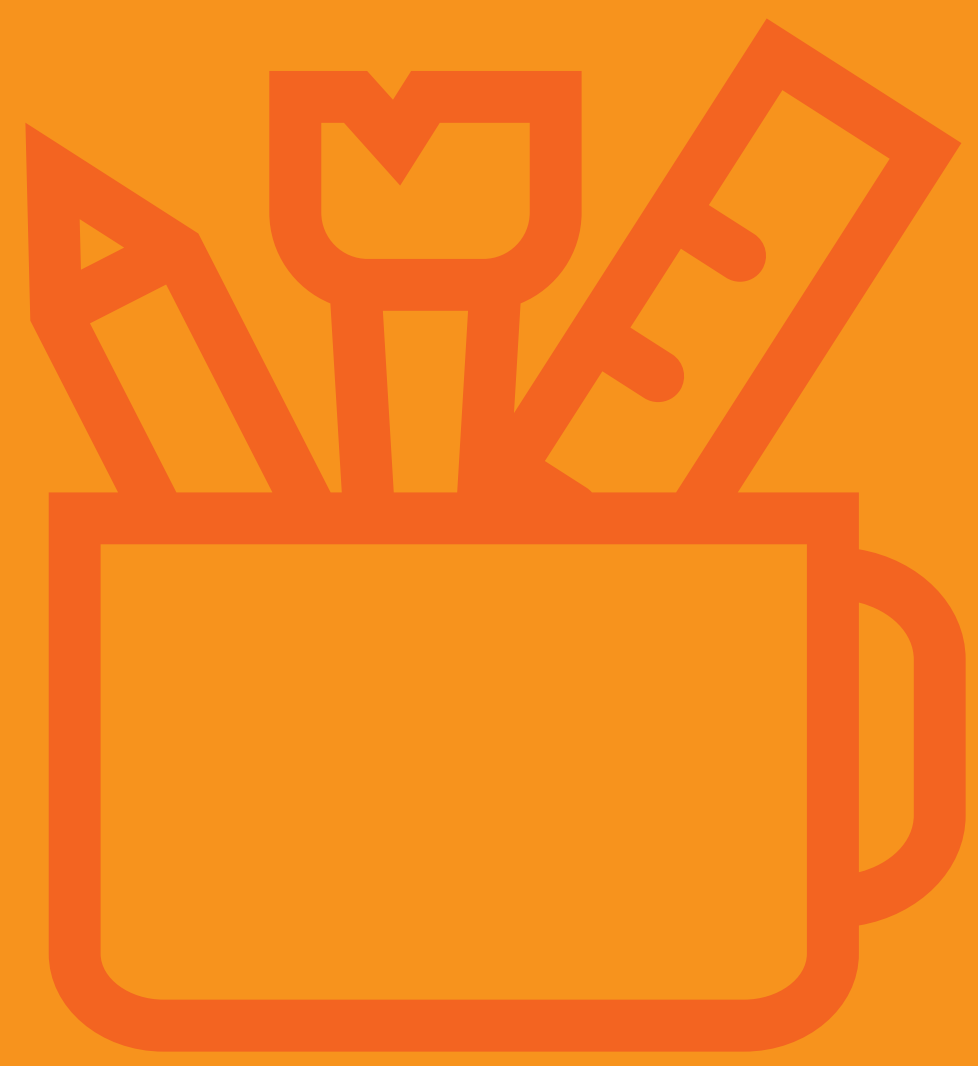

